UMN-D-01-3

hep-th/0011106

\title{
Renormalizing DLCQ Using Supersymmetry
}

\author{
I. Filippov ${ }^{a}{ }^{\text {S. Pinsky, }}{ }^{a}$ and J.R. Hiller ${ }^{b}$ \\ ${ }^{a}$ Department of Physics \\ Ohio State University, Columbus, OH 43210 \\ ${ }^{b}$ Department of Physics \\ University of Minnesota-Duluth, Duluth, MN 55812
}

\begin{abstract}
Recent string theory developments suggest the necessity to understand supersymmetric gauge theories non-perturbatively, in various dimensions. In this work we show that there is a standard Hamiltonian formulation that generates a finite and supersymmetric result at every order of the approximation scheme known as discrete light-cone quantization (DLCQ). We present this renormalized DLCQ Hamiltonian and find that it has two novel features: it automatically chooses the 't Hooft prescription for renormalizing the singularities, and it introduces irrelevant operators that serve to preserve the supersymmetry and improve the convergence. We solve for the bound states and the wave functions with and without the irrelevant operators and verify that with the irrelevant operator the exact large- $N_{c}$ supersymmetric DLCQ (SDLCQ) results are reproduced. With the irrelevant operator removed, we show that the bound-state mass and wave functions appear to be converging to the SDLCQ results but very slowly. This is a first step in extending the advantages of SDLCQ to non-supersymmetric theories.
\end{abstract}

March 2001 


\section{Introduction}

Solving for the non-perturbative properties of quantum field theories - such as QCD - is a very difficult problem. In order to gain some insights, however, a number of lower dimensional models have been investigated in the large- $N_{c}$ (planar) approximation using discrete lightcone quantization (DLCQ), with a plethora of examples appearing over the years (for a review see [1]). Going beyond the simplest models that have either just fermions or just bosons one encounters significant problems.

Recall that in the DLCQ approach, the Schrodinger equation for the field theory is formulated as an infinite set of integral equations for the wave functions and masses of the bound states of the theory. This infinite set of integral equations is then truncated and solved numerically. Problems arise because these integral equations have a number of singularities that need to be regularized and renormalized before any numerical solution can be attempted. In the simplest models one can follow 't Hooft [2] and use the principal value prescription to regulate and effectively renormalize these divergences. There are other prescriptions besides the 't Hooft prescription, and these prescriptions [3], 田] lead to different results. It has only recently been understood that these other prescriptions produce an incomplete solution [5] and that when a complete set of topological components are included they reproduce the 't Hooft prescription. In fact, all the singularities that are encountered in the $(1+1)$-dimensional formulation can be handled by careful analytical calculations and the principal value prescription. We will review these calculations to highlight the difficulties and ambiguities.

A number of recent string theory developments have sharpened the need to understand supersymmetric Yang-Mills non-perturbatively in various dimensions, since they play a crucial role in describing D-brane dynamics, the Maldacena conjecture [6, 0, 8] and, ultimately, in formulating M(atrix) Theory [9]. This makes it imperative to develop a non-perturbative method to solve such theories where fermions and bosons are treated on an equal footing. The importance of supersymmetry in string theory and M-theory is the core of the recent supersymmetric formulation of DLCQ which we call SDLCQ [10]. The principle is to construct a sequence of approximations to the field theory that converge to the continuum theory and that remain supersymmetric at every order of the approximation.

In $1+1$ dimensions it is well known that even $\mathcal{N}=1$ supersymmetric theories are super renormalizable. Therefore a formulation which does not break the symmetry will be totally finite, requiring no regularization or renormalization. In recent years we have solved many such theories and successfully extended these ideas to higher dimensions [11, 12, 13, 14, 15, 16, 17, 18, 19. In this work we would like to return to the original Hamiltonian formulation 
of DLCQ and ask what is the regularization and renormalization that exactly reproduces the SDLCQ formula. The existence of such a formulation has never really been addressed except in a very simple model [15]. In fact, none of the fully supersymmetric theories that we have solved over the last few years have been solved using the standard Hamiltonian DLCQ method (or by any other method) because of the complexities we mentioned above. We will show that there is a regular and renormalizable Hamiltonian formulation that exactly reproduces the results of SDLCQ at large $N_{c}$, and we will see that the principal value prescription is a natural consequence of SDLCQ. Since SDLCQ has been shown to produce finite results in higher dimensions, these results imply that SDLCQ can be used to generate finite Hamiltonians in higher dimensions as well.

We will also find that the SDLCQ Hamiltonian contains a number of irrelevant operators which we call kronecker terms. These kronecker terms serve to maintain the supersymmetry at every order of the approximation and act as convergence factors. If we remove the kronecker terms and calculate the bound-state masses and wave functions, we find that, while supersymmetry is now broken at every order, the states very slowly converge to the supersymmetric bound states of SDLCQ.

We should stress that in the model we study here we compactify the null direction $x^{-}$, and we drop the zero-mode sector, which is conventional in DLCQ. We will argue that dropping some of the zero modes should not be viewed as an omission but rather as the renormalization subtraction that produces the 't Hooft principal value prescription and supersymmetry.

This paper is organized as follows. In section 2 we review some of the complexities that one finds in theories with dynamical bosons and fermions even in $1+1$ dimensions. In section 3 we review SDLCQ for $\mathcal{N}=1$ super Yang-Mills in $1+1$ dimensions, and in section 4 we present the DLCQ Hamiltonian for this theory that numerically exactly preserves supersymmetry and discuss the emergence of the principal value prescription and kronecker terms and our numerical results. We end with some discussion of these results and the implications for future work in section 5 .

\section{Complexities of DLCQ}

Very few DLCQ calculations involving both dynamical fermions and bosons have been performed even in 1+1 dimensions because of complexities associated with renormalization.1 We will briefly review these issues. For a more complete discussion the reader is referred to Ref. [20].

\footnotetext{
${ }^{1}$ For recent work on Pauli-Villars regularization in DLCQ, see Ref. [21.
} 
The instantaneous Coulomb interactions involving $2 \rightarrow 2$ parton interactions behave singularly when there is an exchange of zero momentum. The same type of Coulomb singularity involving $2 \rightarrow 2$ boson-boson interactions appears in much simpler models [22], and can be shown to cancel a 'self-induced' mass term (or self-energy) obtained from normal ordering the Hamiltonian. The same prescription works in the models involving fermions and bosons. There are, however, finite residual terms after this cancellation is explicitly performed for the boson-boson and boson-fermion interactions, and they cannot be absorbed by a redefinition of existing coupling constants. These residual terms behave as momentum-dependent mass terms, and the momentum dependence is not uniquely determined. Examples of these terms can be found in Ref. [20]; they simply multiply the wave functions in the bound-state integral equations.

When one integrates out the left-handed fermion fields, which are dependent variables (satisfying an equation of the form $\partial_{-} \psi_{L}=F$ ) in light-cone quantization, there appears in the light-cone Hamiltonian a contribution of the form $F^{\dagger} \frac{1}{\mathrm{i} \partial_{-}} F$, which is singular at $k^{+}=0$. This singularity is canceled by a (divergent) momentum-dependent mass term that comes from normal ordering the $F^{\dagger} \frac{1}{\mathrm{i} \partial_{-}} F$ interactions and performing an appropriate (infinite) renormalization of the bare boson mass. The mechanism for cancellation here is different from the Coulombic case, since it requires specific endpoint relations for wave functions. For example, the bound-state integral equation governing the behavior of the two-particle wave function $f\left(k_{1}, k_{2}\right)$ and the three-particle wave function $h\left(k_{1}, k_{2}, k_{3}\right)$ is singular for vanishing longitudinal momentum fraction in the three-particle wave function. However, these divergences are precisely canceled by the momentum-dependent mass terms. To see this, one must consider the integral equation governing the three-parton wave function $h\left(k_{1}, k_{2}, k_{3}\right)$ at zero momentum fraction. This leads to the "ladder relations" $h\left(0, x_{2}, x_{3}\right) \propto f\left(x_{2}, x_{3}\right) / \sqrt{x_{2}}$ and $h\left(x_{1}, 0, x_{3}\right) \propto f\left(x_{1}, x_{3}\right) / \sqrt{x_{1}}$. The name "ladder relations" refers to the fact that they are relations between wave functions with different numbers of partons. It can then be shown that the singular behavior of the integral involving the wave function $h$ can be written in terms of a momentum-dependent mass term involving the wave function $f$ by virtue of corresponding "ladder relations" [23]. The sum of these divergent contributions exactly cancels the self-energy contribution. These cancellations generalize to all the integral equations.

This discussion gives a sense of the difficulties encountered in setting up DLCQ in nontrivial theories involving both fermions and bosons even in $1+1$ dimensions. What we will see in the following sections is that SDLCQ gives a regularization and renormalization that automatically provides the cancellation of the self-induced mass and the Coulomb singularity, and there is no need for the delicate cancellation through ladder relations. In fact, we will see that every term in the Hamiltonian is finite by itself, and no conditions or constraints are needed to obtain this Hamiltonian beyond the SDLCQ formulation. We will see that 
even the 't Hooft principal value prescription naturally follows from SDLCQ.

\section{Formulation of the bound state problem.}

The light-cone formulation of the supersymmetric matrix model obtained by dimensionally reducing $\mathcal{N}=1 \mathrm{SYM}_{2+1}$ to $1+1$ dimensions has already appeared in [10], to which we refer the reader for explicit derivations. We simply note here that the light-cone Hamiltonian $P^{-}$ is given in terms of the supercharge $Q^{-}$via the supersymmetry relation $\left\{Q^{-}, Q^{-}\right\}=2 \sqrt{2} P^{-}$, where

$$
Q^{-}=2^{3 / 4} g \int d x^{-} \operatorname{tr}\left\{\left(\mathrm{i}\left[\phi, \partial_{-} \phi\right]+2 \psi \psi\right) \frac{1}{\partial_{-}} \psi\right\}
$$

In the above, $\phi_{i j}=\phi_{i j}\left(x^{+}, x^{-}\right)$and $\psi_{i j}=\psi_{i j}\left(x^{+}, x^{-}\right)$are $N_{c} \times N_{c}$ Hermitian matrix fields representing the physical boson and fermion degrees of freedom (respectively) of the theory. These fields are remnants of the physical transverse degrees of freedom of the original $2+1$ dimensional theory. All unphysical degrees of freedom present in the original Lagrangian have been explicitly eliminated. There are no ghosts. This is a special feature of light-cone quantization in light-cone gauge.

For completeness, we indicate the additional relation $\left\{Q^{+}, Q^{+}\right\}=2 \sqrt{2} P^{+}$for the lightcone momentum $P^{+}$, where

$$
Q^{+}=2^{1 / 4} \int d x^{-} \operatorname{tr}\left[\left(\partial_{-} \phi\right)^{2}+\mathrm{i} \psi \partial_{-} \psi\right]
$$

The $(1,1)$ supersymmetry of the model follows from the fact $\left\{Q^{+}, Q^{-}\right\}=0$. In order to quantize $\phi$ and $\psi$ on the light cone, we first introduce the following expansions at fixed light-cone time $x^{+}=0$ :

$$
\begin{array}{r}
\phi_{i j}\left(x^{-}, 0\right)=\frac{1}{\sqrt{2 \pi}} \int_{0}^{\infty} \frac{d k^{+}}{\sqrt{2 k^{+}}}\left(a_{i j}\left(k^{+}\right) e^{-i k^{+} x^{-}}+a_{j i}^{\dagger}\left(k^{+}\right) e^{i k^{+} x^{-}}\right), \\
\psi_{i j}\left(x^{-}, 0\right)=\frac{1}{2 \sqrt{\pi}} \int_{0}^{\infty} d k^{+}\left(b_{i j}\left(k^{+}\right) e^{-i k^{+} x^{-}}+b_{j i}^{\dagger}\left(k^{+}\right) e^{i k^{+} x^{-}}\right) .
\end{array}
$$

We then specify the commutation relations

$$
\left[a_{i j}\left(p^{+}\right), a_{l k}^{\dagger}\left(q^{+}\right)\right]=\left\{b_{i j}\left(p^{+}\right), b_{l k}^{\dagger}\left(q^{+}\right)\right\}=\delta\left(p^{+}-q^{+}\right) \delta_{i l} \delta_{j k}
$$

for the gauge group $\mathrm{U}\left(N_{c}\right)$, or $\mathrm{SU}\left(N_{c}\right)$ in large $N_{c}$.

For the bound-state eigen-problem $2 P^{+} P^{-}\left|\Psi>=M^{2}\right| \Psi>$, we may restrict the subspace of states to those with fixed light-cone momentum $P^{+}$, on which $P^{+}$is diagonal, and so the bound-state problem is reduced to the diagonalization of the light-cone Hamiltonian $P^{-}$. 
Since $P^{-}$is proportional to the square of the supercharge $Q^{-}$, any eigenstate $\mid \Psi>$ of $P^{-}$ with mass squared $M^{2}$ gives rise to a natural four-fold degeneracy in the spectrum because of the supersymmetry algebra - all four states below have the same mass:

$$
\left|\Psi>, \quad Q^{+}\right| \Psi>, \quad Q^{-}\left|\Psi>, \quad Q^{+} Q^{-}\right| \Psi>
$$

Although this four-fold degeneracy is realized in the continuum formulation of the theory, this property will not necessarily survive if we choose to discretize the theory in an arbitrary manner. However, an important feature of SDLCQ is that it does preserve the exact four-fold degeneracy for any resolution.

The explicit equation for $Q^{-}$, in the momentum representation, is obtained by substituting the quantized field expressions (3) and (4) directly into the definition of the supercharge (四). The result is:

$$
\begin{aligned}
Q^{-}= & \frac{\mathrm{i} 2^{-1 / 4} g}{\sqrt{\pi}} \int_{0}^{\infty} d k_{1} d k_{2} d k_{3} \delta\left(k_{1}+k_{2}-k_{3}\right)\{ \\
& \frac{1}{2 \sqrt{k_{1} k_{2}}} \frac{k_{2}-k_{1}}{k_{3}}\left[a_{i k}^{\dagger}\left(k_{1}\right) a_{k j}^{\dagger}\left(k_{2}\right) b_{i j}\left(k_{3}\right)-b_{i j}^{\dagger}\left(k_{3}\right) a_{i k}\left(k_{1}\right) a_{k j}\left(k_{2}\right)\right] \\
& \frac{1}{2 \sqrt{k_{1} k_{3}}} \frac{k_{1}+k_{3}}{k_{2}}\left[a_{i k}^{\dagger}\left(k_{3}\right) a_{k j}\left(k_{1}\right) b_{i j}\left(k_{2}\right)-a_{i k}^{\dagger}\left(k_{1}\right) b_{k j}^{\dagger}\left(k_{2}\right) a_{i j}\left(k_{3}\right)\right] \\
& \frac{1}{2 \sqrt{k_{2} k_{3}}} \frac{k_{2}+k_{3}}{k_{1}}\left[b_{i k}^{\dagger}\left(k_{1}\right) a_{k j}^{\dagger}\left(k_{2}\right) a_{i j}\left(k_{3}\right)-a_{i j}^{\dagger}\left(k_{3}\right) b_{i k}\left(k_{1}\right) a_{k j}\left(k_{2}\right)\right] \\
& \left.\left(\frac{1}{k_{1}}+\frac{1}{k_{2}}-\frac{1}{k_{3}}\right)\left[b_{i k}^{\dagger}\left(k_{1}\right) b_{k j}^{\dagger}\left(k_{2}\right) b_{i j}\left(k_{3}\right)+b_{i j}^{\dagger}\left(k_{3}\right) b_{i k}\left(k_{1}\right) b_{k j}\left(k_{2}\right)\right]\right\} .
\end{aligned}
$$

In order to implement the DLCQ formulation [1] of the theory, we simply restrict the momenta $k_{1}, k_{2}$ and $k_{3}$ appearing in the above equation to the following set of allowed momenta: $\left\{\frac{P^{+}}{K}, \frac{2 P^{+}}{K}, \frac{3 P^{+}}{K}, \ldots\right\}$. Here $K$ is some arbitrary positive integer and must be sent to infinity if we wish to recover the continuum formulation of the theory. The integer $K$ is called the harmonic resolution, and $1 / K$ measures the coarseness of our discretization. Physically, $1 / K$ represents the smallest unit of longitudinal momentum fraction allowed for each parton. As soon as we implement the DLCQ procedure, which is specified unambiguously by the harmonic resolution $K$, the integrals appearing in the definition of $Q^{-}$are replaced by finite sums, and the eigen-equation is reduced to a finite matrix problem. In this discrete formulation all operators containing a zero-momentum operator $a_{i j}(0), a_{i j}^{\dagger}(0), b_{i j}(0)$ or $b_{i j}^{\dagger}(0)$ are dropped. We discuss the consequences of this below. 


\section{Hamiltonian Regularization}

In this section we will present the DLCQ Hamiltonian that exactly reproduces SDLCQ in the large- $N_{c}$ limit and which is therefore totally renormalized. We will use a standard operator ordering and suppress all the indices and variables as follows:

$$
\begin{aligned}
a^{\dagger} a & =a_{i j}^{\dagger}(k) a_{i j}(k), \\
a^{\dagger} a^{\dagger} a a & =a_{i j}^{\dagger}\left(k_{3}\right) a_{j s}^{\dagger}\left(k_{4}\right) a_{i p}\left(k_{1}\right) a_{p s}\left(k_{2}\right) \delta\left(k_{1}+k_{2}-k_{3}-k_{4}\right), \\
a^{\dagger} a a a & =a_{i j}^{\dagger}\left(k_{4}\right) a_{i s}\left(k_{1}\right) a_{s p}\left(k_{2}\right) a_{p j}\left(k_{3}\right) \delta\left(k_{1}+k_{2}+k_{3}-k_{4}\right), \\
a^{\dagger} a^{\dagger} a^{\dagger} a & =a_{i j}^{\dagger}\left(k_{1}\right) a_{j s}^{\dagger}\left(k_{2}\right) a_{s p}^{\dagger}\left(k_{3}\right) a_{i p}\left(k_{4}\right) \delta\left(k_{1}+k_{2}+k_{3}-k_{4}\right) .
\end{aligned}
$$

There is a well defined and unambiguous method to find the DLCQ Hamiltonian. We start from the discrete SDLCQ supercharge, square it, and then normal order the results. In the continuum formulation this is, of course, a trivial restatement that the Hamiltonian is the square of the supercharge; however, it is not a trivial statement in the discrete formulation since the zero-mode operators have been dropped. In the normal ordering process one contracts various operators to form the Hamiltonian operator, but in SDLCQ the zero-mode operators are missing, and, therefore, the Hamiltonian will be missing operators that would have been formed from the contraction of the zero modes. In addition, of course, the zero modes that are normally dropped in DLCQ are also dropped here. After considerable algebra one arrives at the normal ordered form of the square of the discrete supercharge, which is our renormalized DLCQ Hamiltonian

$$
\begin{aligned}
P^{-} & =\frac{g^{2} N_{c}}{4 \pi} \int_{0}^{\infty} d k_{1} \frac{\mu^{2}\left(k_{1}\right)}{k_{1}}\left(a^{\dagger} a+b^{\dagger} b\right)+\frac{g^{2}}{4 \pi} \int_{0}^{\infty} d k_{1} d k_{2} d k_{3} d k_{4}[ \\
& +A_{1} b^{\dagger} b^{\dagger} b b+A_{2}\left(b^{\dagger} b b b-b^{\dagger} b^{\dagger} b^{\dagger} b\right)+B_{1} a^{\dagger} a^{\dagger} a a+B_{2}\left(a^{\dagger} a a a+a^{\dagger} a^{\dagger} a^{\dagger} a\right) \\
& +C_{1} b^{\dagger} b^{\dagger} a a+C_{2} a^{\dagger} a^{\dagger} b b+C_{3} b^{\dagger} a^{\dagger} b a+C_{4} a^{\dagger} b^{\dagger} a b+C_{5} b^{\dagger} a^{\dagger} a b+C_{6} a^{\dagger} b^{\dagger} b a \\
& +D_{1}\left(a^{\dagger} a b b-a^{\dagger} b^{\dagger} b^{\dagger} a\right)+D_{2}\left(a^{\dagger} b a b-b^{\dagger} a^{\dagger} b^{\dagger} a\right)+D_{3}\left(a^{\dagger} b b a-b^{\dagger} b^{\dagger} a^{\dagger} a\right) \\
& \left.+D_{4}\left(b^{\dagger} b a a+b^{\dagger} a^{\dagger} a^{\dagger} b\right)+D_{5}\left(b^{\dagger} a b a+a^{\dagger} b^{\dagger} a^{\dagger} b\right)+D_{6}\left(b^{\dagger} a a b+a^{\dagger} a^{\dagger} b^{\dagger} b\right)\right]
\end{aligned}
$$

where

$$
\mu^{2}\left(k_{1}\right)=\int_{0}^{k_{1}} d k_{2} \frac{\left(k_{1}+k_{2}\right)^{2}}{k_{2}\left(k_{1}-k_{2}\right)^{2}},
$$

and the other coefficients are given by

$$
A_{1}=P V \frac{2}{\left(k_{4}-k_{2}\right)^{2}}-\frac{2}{\left(k_{1}+k_{2}\right)^{2}}-\delta_{1,3}\left(\frac{2}{k_{1}^{2}}+\frac{2}{k_{2}^{2}}\right),
$$




$$
\begin{aligned}
& A_{2}=\frac{2}{\left(k_{2}+k_{3}\right)^{2}}-\frac{2}{\left(k_{1}+k_{2}\right)^{2}}, \\
& B_{1}=\frac{1}{\sqrt{4 k_{1} k_{2} k_{3} k_{4}}}\left(\frac{\left(k_{1}-k_{2}\right)\left(k_{3}-k_{4}\right)}{\left(k_{1}+k_{2}\right)^{2}}-P V \frac{\left(k_{1}+k_{3}\right)\left(k_{2}+k_{4}\right)}{\left(k_{4}-k_{2}\right)^{2}}\right) \text {, } \\
& B_{2}=\frac{1}{\sqrt{4 k_{1} k_{2} k_{3} k_{4}}}\left(\frac{\left(k_{3}-k_{2}\right)\left(k_{1}+k_{4}\right)}{\left(k_{3}+k_{2}\right)^{2}}+\frac{\left(k_{1}-k_{2}\right)\left(k_{3}+k_{4}\right)}{\left(k_{1}+k_{2}\right)^{2}}\right), \\
& C_{1}=\frac{1}{\sqrt{k_{1} k_{2}}}\left(\frac{k_{1}-k_{2}}{\left(k_{1}+k_{2}\right)^{2}}+P V \frac{1}{2\left(k_{3}-k_{1}\right)}-\frac{\left(k_{1}-k_{2}\right) \delta_{1,3}}{k_{3} k_{4}}\right) \text {, } \\
& C_{2}=\frac{1}{\sqrt{k_{3} k_{4}}}\left(\frac{k_{4}-k_{3}}{\left(k_{1}+k_{2}\right)^{2}}+P V \frac{1}{2\left(k_{3}-k_{1}\right)}-\frac{\left(k_{4}-k_{3}\right) \delta_{1,3}}{k_{1} k_{2}}\right) \text {, } \\
& C_{3}=-\frac{1}{\sqrt{k_{2} k_{4}}}\left(P V \frac{k_{2}+k_{4}}{\left(k_{2}-k_{4}\right)^{2}}-\frac{1}{2\left(k_{1}+k_{2}\right)}-\frac{2 k_{4} \delta_{1,3}}{k_{1}^{2}}\right), \\
& C_{4}=-\frac{1}{\sqrt{k_{1} k_{3}}}\left(P V \frac{k_{1}+k_{3}}{\left(k_{1}-k_{3}\right)^{2}}-\frac{1}{2\left(k_{1}+k_{2}\right)}-\frac{2 k_{1} \delta_{1,3}}{k_{2}^{2}}\right) \text {, } \\
& C_{5}=-\frac{1}{\sqrt{4 k_{1} k_{4}}}\left(P V \frac{1}{\left(k_{3}-k_{1}\right)}+\frac{1}{\left(k_{1}+k_{2}\right)}+\frac{2\left(k_{1}+k_{2}\right) \delta_{1,3}}{k_{1} k_{2}}\right) \text {, } \\
& C_{6}=-\frac{1}{\sqrt{4 k_{2} k_{3}}}\left(-P V \frac{1}{\left(k_{3}-k_{1}\right)}+\frac{1}{\left(k_{1}+k_{2}\right)}+\frac{2\left(k_{1}+k_{2}\right) \delta_{1,3}}{k_{2} k_{3}}\right) \text {, } \\
& D_{1}=\frac{1}{\sqrt{k_{1} k_{4}}}\left(\frac{k_{1}+k_{4}}{\left(k_{2}+k_{3}\right)^{2}}-\frac{1}{2\left(k_{1}+k_{2}\right)}\right) \text {, } \\
& D_{2}=-\frac{1}{\sqrt{4 k_{2} k_{4}}}\left(\frac{1}{\left(k_{2}+k_{3}\right)}-\frac{1}{\left(k_{1}+k_{2}\right)}\right) \text {, } \\
& D_{3}=-\frac{1}{\sqrt{k_{3} k_{4}}}\left(\frac{k_{3}+k_{4}}{\left(k_{1}+k_{2}\right)^{2}}-\frac{1}{2\left(k_{2}+k_{3}\right)}\right) \text {, } \\
& D_{4}=\frac{1}{\sqrt{k_{2} k_{3}}}\left(\frac{k_{3}-k_{2}}{\left(k_{2}+k_{3}\right)^{2}}+\frac{1}{2\left(k_{1}+k_{2}\right)}\right) \text {, } \\
& D_{5}=-\frac{1}{\sqrt{4 k_{1} k_{3}}}\left(\frac{1}{\left(k_{2}+k_{3}\right)}+\frac{1}{\left(k_{1}+k_{2}\right)}\right) \text {, } \\
& D_{6}=-\frac{1}{\sqrt{k_{1} k_{2}}}\left(\frac{k_{2}-k_{1}}{\left(k_{1}+k_{2}\right)^{2}}-\frac{1}{2\left(k_{2}+k_{3}\right)}\right) \text {. }
\end{aligned}
$$

It is understood that the integrals become finite sums for the DLCQ calculation. The forms of the counterterms are included in the coefficients, $A_{i}, B_{i}, C_{i}$, and $D_{i}$.

In comparing this result to SDLCQ, the first obvious feature is that the Hamiltonian has many more terms than the supercharge. Numerically this is quite significant since each of these terms has to act on the entire fock space to calculate the Hamiltonian matrix, and, therefore, the DLCQ approach is more time consuming. In SDLCQ the supercharge must be squared before it is used to calculate the spectrum; however, squaring a numerical matrix 
can be done very efficiently.

The second thing to notice is the appearance of the principal value regularization of the singularities in terms $A_{1}, B_{1}$ and all the $C_{i}$ terms. The use of the principal value is a common feature in DLCQ dating back to the 't Hooft model [2]. What is new here is that, if we trace the origin of these subtractions back to the SDLCQ formulation, we see that they occur because we dropped the zero-mode operators. The operators that are discarded by the principal value prescription are just the set of operators that appear when normal ordering the zero modes. In the discrete calculation we include theta functions ${ }^{2}$ to enforce the missing zero-mode contributions. We always find two identical terms that are missing the zero-mode contribution, but one comes with a $\theta\left(k_{3}-k_{1}\right)$ and while the other comes with $\theta\left(k_{1}-k_{3}\right)$. In the continuum limit these combine to one, but in the discrete calculation they combine to give the principal value. While normally one considers dropping any mode in a calculation an undesirable approximation, here we see that dropping zero modes is equivalent to a renormalization subtraction and, in fact, an unexpectedly good subtraction.

Some time ago another method of treating this singularity, which produced a different numerical result, was suggested by $\mathrm{Wu}$ [3] and by Mandelstam and Leibbrandt [4]. It is only recently that the connection between this subtraction and the principal value 't Hooft prescription was fully understood. It was shown in [5] that, in fact, the 't Hooft prescription is equivalent to an infinite set of topological terms in the other prescriptions. The fact that subtracting the intermediate zero modes in SDLCQ automatically leads one to this correct result is clearly one of the attractive features of this method.

Another important feature of this Hamiltonian, that one would not see in the usual DLCQ Hamiltonian, are the terms with the kronecker delta. These terms, which we call kronecker terms, are zero in the continuum formulation and scale to zero as the resolution gets large in the DLCQ formulation. These terms arise in the discrete calculation from terms of the form $1-\left(\theta\left(k_{1}-k_{3}\right)+\theta\left(k_{3}-k_{1}\right)\right)$. They destroy two particles and replace them with two particles with the same momentum. The coefficients include a momentum dependent factor that scales to zero as the resolution goes to infinity.t

To fully understand the significance of these kronecker terms, it is helpful to write one of them in discrete form. Without any leading numerical factors the $A_{1}$ term in the Hamiltonian has the following discrete form:

$$
P^{-} \propto L g^{2} \sum_{n_{1}, n_{2}, n_{3}, n_{4}=1, n_{4} \neq n_{2}}^{\infty} B^{\dagger}\left(n_{3}\right) B^{\dagger}\left(n_{4}\right) B\left(n_{1}\right) B\left(n_{2}\right) \frac{2}{\left(n_{4}-n_{2}\right)^{2}} \delta_{n_{1}+n_{2}-n_{3}-n_{4}}
$$

\footnotetext{
${ }^{2} \theta(x)=1$ for $x>0$ and zero otherwise.

${ }^{3}$ We are thinking of the integrals as sums when we write these terms as kronecker deltas.

${ }^{4}$ For earlier work on this type of zero-mode contribution, see Ref. [24].
} 


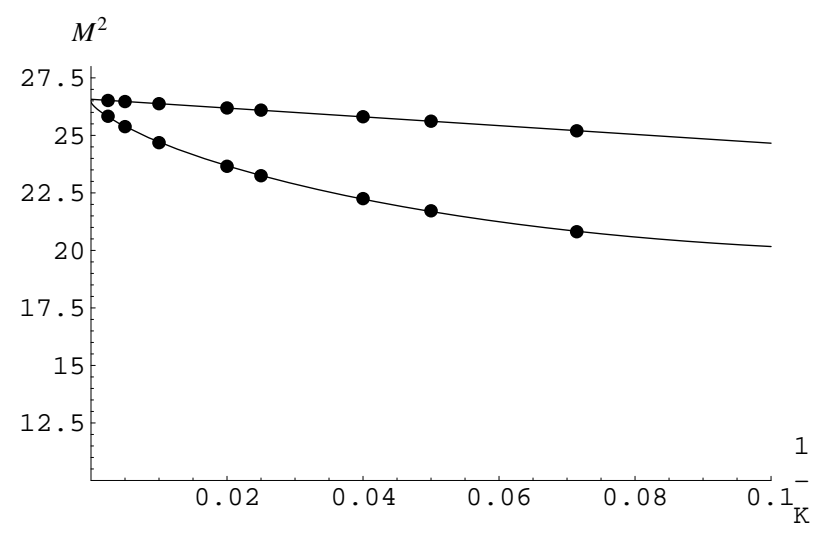

(a)

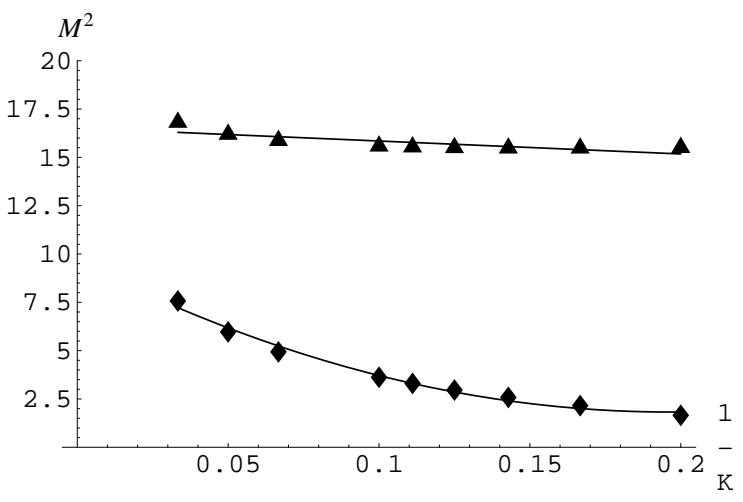

(b)

Figure 1: $\frac{\pi M^{2}}{g^{2} N_{c}}$ vs. $\frac{1}{K}$, where $K$ is the resolution, for (a) the Kutasov model and (b) full $\mathcal{N}=1$ SYM. In each case the upper curve includes the kronecker terms, but the lower one does not.

$$
\begin{aligned}
& -L g^{2} \sum_{n_{1}, n_{2}, n_{3}, n_{4}=1}^{\infty} B^{\dagger}\left(n_{3}\right) B^{\dagger}\left(n_{4}\right) B\left(n_{1}\right) B\left(n_{2}\right) \frac{2}{\left(n_{1}+n_{2}\right)^{2}} \delta_{n_{1}+n_{2}-n_{3}-n_{4}} \\
& \text { - } L g^{2} \sum_{n_{1}, n_{2}=1}^{\infty} B^{\dagger}\left(n_{1}\right) B^{\dagger}\left(n_{2}\right) B\left(n_{1}\right) B\left(n_{2}\right)\left(\frac{2}{\left(n_{1}\right)^{2}}+\frac{2}{\left(n_{2}\right)^{2}}\right) .
\end{aligned}
$$

In this form only $g$ and $L$ carry dimensions. Now let us go to infinite resolution and convert the sums to integrals. The detailed translation between the discrete and continuous formulation is given in Ref. [10]. For our purposes it is sufficient to note that

$$
k_{i}=\frac{n_{i} \pi}{L}, \quad P^{+}=\frac{K \pi}{L}, \quad \frac{\pi}{L} \sum_{n=1}^{\infty} \rightarrow \int_{0}^{\infty}, \quad B(n) \rightarrow \sqrt{\frac{\pi}{L}} b(n) .
$$

Then the contribution of this term to $P^{-}$translates to

$$
\begin{aligned}
P^{-} \propto & P V \int_{0}^{\infty} \prod_{i=1}^{4} d k_{i} \delta\left(k_{1}+k_{2}-k_{3}-k_{4}\right) b^{\dagger}\left(k_{3}\right) b^{\dagger}\left(k_{4}\right) b\left(k_{1}\right) b\left(k_{4}\right)\left(\frac{2}{\left(k_{4}-k_{2}\right)^{2}}-\frac{2}{\left(k_{1}+k_{2}\right)^{2}}\right) \\
& +\frac{P^{+}}{K} \int_{0}^{\infty} d k_{1} d k_{2} b^{\dagger}\left(k_{1}\right) b^{\dagger}\left(k_{2}\right) b\left(k_{1}\right) b\left(k_{2}\right)\left(\frac{2}{\left(k_{1}\right)^{2}}+\frac{2}{\left(k_{2}\right)^{2}}\right) .
\end{aligned}
$$

The appearance of $1 / K$ in the second term shows that this term is irrelevant. As $K$ goes to infinity this term goes away.

We first saw this type of term in the SDLCQ formulation [15 of a supersymmetric model proposed by Kutasov 25]. The Kutasov model is essentially the above Hamiltonian with an appropriately chosen mass term. The kronecker terms serve two functions; they serve to enforce the supersymmetry at every resolution and act as convergence factors. Dropping these terms, one gets the same numerical results at infinite resolution, but the convergence 
is very slow, and supersymmetry is broken at finite resolution. In addition, massless states are driven to negative $M^{2}$ values. In Fig. 17a we show our results for the Kutasov model [15]. The top flat curve is SDLCQ while the lower curve is the result when the kronecker terms are removed. In this calculation we had to go to very high resolution to actually see the two different versions of the theory converge [11]. We see very similar results in Fig. 10 for the full SYM theory. We find that the effect of the kronecker terms is very large and the convergence is very slow without these terms. We cannot go to high enough resolution to obtain the complete convergence seen in the Kutasov model.

To get to these very high resolutions in either model we had to truncate the fock space [15]. To get to the highest resolution in the full SYM theory we truncated the basis to five particles. We have compared the effect of this truncation up to resolution $K=10$ and find it leads to only small changes in the mass of the bound state. There are many bound states in the region of this bound state that we do not show. To make sure we are referring to the same state in the two calculations we take the inner product of the wave functions. We find that wave functions in the two calculations have better than a $90 \%$ overlap. This also gives us information about the effect on the wave functions of dropping the kronecker terms.

This renormalized Hamiltonian is a starting point to begin the investigation of nonsupersymmetric theories that cannot be written as the square of a supercharge. In addition there already exist SDLCQ calculations in $2+1$ dimensions [14, 26] which can be used to produce non-perturbatively renormalized Hamiltonians in $2+1$ dimensions.

Finally, we should remind the reader that there is a set of zero modes that we have not addressed here, the diagonal zero modes of $A^{+}$and its superpartners. These modes are discussed elsewhere [17]. They give rise to modes that wind around the compact space and to the $N_{c}$ degenerate vacua of this model.

\section{Conclusions}

The non-perturbative renormalization of a light-cone quantized Hamiltonian gauge theory with dynamical bosons and fermions can be a complicated and ambiguous procedure even in $1+1$ dimensions. As a result there have only been a few DLCQ calculations of this type. A promising approach appears to be the very natural marriage of DLCQ with supersymmetry. Together they generate a powerful numerical technique, SDLCQ, for solving exactly supersymmetric theories. To date many exactly supersymmetric theories in $1+1$ [19] and $2+1$ 14, 26, 27 dimensions have been solved using SDLCQ, and the results of these calculations have been used to address a number of fundamental issues in string theory and related areas. 
In this paper we revisited DLCQ and found the DLCQ Hamiltonian that exactly preserve supersymmetry. We present a procedure for producing non-perturbative renormalized DLCQ Hamiltonians that are free from the complexities that one normally encounters in DLCQ. We found a unique set of counterterms in DLCQ that achieve this result and that have a number of important properties. Surprisingly we discovered that dropping zero modes in SDLCQ should be viewed as the renormalization subtraction that produces the 't Hooft principal value prescription in DLCQ. This is particularly appealing since the principal value prescription has recently been shown [5] to automatically include a series of topological corrections not included in other prescriptions [3, 4].

In addition we find a set of irrelevant terms, which we call kronecker terms, that scale away at infinite resolution. They make the Hamiltonian exactly supersymmetric at every resolution and serve as convergence factors. The importance of numerical convergence factors should not be overlooked; they can be the difference between a successful calculation and one that has to await larger and faster computers. We presented results for $\mathcal{N}=1 \mathrm{SYM}$ models with and without the kronecker terms and found that they have a large effect and that the convergence without kronecker terms is very slow. When we include the kronecker terms, the large- $N_{c}$ SDLCQ results for the Kutasov model match those of Ref. [15].

This improved technology represents a first step toward extending the advantages of SDLCQ to DLCQ and treating models with supersymmetry breaking. There already exist SDLCQ calculations in 2+1 dimensions [14, 26, 27] which can be used to produce nonperturbatively renormalized Hamiltonians in $2+1$ dimensions, and we hope to use this new renormalization technique to study theories that break supersymmetry.

\section{Acknowledgments}

This work was supported in part by the US Department of Energy. One of the authors (S.P) would like to acknowledge the hospitality of the Aspen Center of Physics where part of the work was completed. The authors would like to acknowledge conversations with U. Trittmann and O. Lunin.

\section{References}

[1] S.J. Brodsky, H.C. Pauli, and S.S. Pinsky, Phys. Rep. 301 (1998) 299, hep-ph/9705477.

[2] G. 't Hooft, Nucl. Phys. B75 (1974) 461. 
[3] T.T. Wu, Phys. Lett. 71B (1977) 142.

[4] S. Mandelstam, Nucl. Phys. B213 (1983) 149; G. Leibbrandt, Phys. Rev. D29 (1984) 1699.

[5] A. Bassetto, F. Vian, and L. Griguolo, Nucl. Phys. Proc. Suppl. B90 (2000) 61, hep-th/0008176; A. Bassetto, L. Griguolo, and F. Vian, Nucl. Phys. B559 (1999) 563, hep-th/9906125.

[6] J. Maldacena, Adv. Theor. Math. Phys. 2 (1998) 231, hep-th/9711200.

[7] F. Antonuccio, O. Lunin, S. Pinsky, and A. Hashimoto, JHEP 07 (1999) 029.

[8] J.R. Hiller, O. Lunin, S. Pinsky, and U. Trittmann, Phys. Lett. B482 (2000) 409, hep-th/0003249.

[9] T. Banks, W. Fischler, S. Shenker, and L. Susskind, Phys. Rev. D55 (1997) 5112, hep-th/9610043.

[10] Y. Matsumura, N. Sakai, and T. Sakai, Phys. Rev. D52 (1995) 2446, hep-th/9504150.

[11] F. Antonuccio, O. Lunin, and S. Pinsky, Phys. Lett. B429 (1998) 327, hep-th/9803027.

[12] F. Antonuccio, O. Lunin, and S. Pinsky, Phys. Rev. D58 (1998) 085009, hep-th/9803170.

[13] F. Antonuccio, O. Lunin, and S. Pinsky, Phys. Lett. B442 (1998) 173, hep-th/9809165.

[14] F. Antonuccio, O. Lunin, and S. Pinsky, Phys. Rev. D59 (1999) 085001, hep-th/9811083.

[15] F. Antonuccio, O. Lunin, and S. Pinsky, Phys. Lett. B459 (1999) 159, hep-th/9904027.

[16] F. Antonuccio, O. Lunin, S. Pinsky, H. C. Pauli, and S. Tsujimaru, Phys. Rev. D58 (1998) 105024, hep-th/9806133.

[17] F. Antonuccio, O. Lunin, S. Pinsky, and S. Tsujimaru, Phys. Rev. D60 (1999) 115006, hep-th/9811254.

[18] F. Antonuccio, H. C. Pauli, S. Pinsky, and S. Tsujimaru, Phys. Rev. D58 (1998) 125006, hep-th/9808120. 
[19] O. Lunin and S. Pinsky, in the proceedings of 11th International Light-Cone School and Workshop: New Directions in Quantum Chromodynamics and 12th Nuclear Physics Summer School and Symposium (NuSS 99), Seoul, Korea, 26 May - 26 Jun 1999 (New York, AIP, 1999), p. 140, hep-th/9910222.

[20] F. Antonuccio and S.S. Pinsky, Phys. Lett. B397 (1997) 42, hep-th/9612021.

[21] S.J. Brodsky, J.R. Hiller, and G. McCartor, Phys. Rev. D58 (1998) 025005, hep-th/9802120; D60 (1999) 054506, hep-ph/9903388.

[22] K. Demeterfi, I.R. Klebanov, and G. Bhanot, Nucl. Phys. B418 (1994) 15.

[23] F. Antonuccio, S.J. Brodsky, and S. Dalley, Phys. Lett. B412 (1997) 104, hep-th/9705413.

[24] J.J. Wivoda and J.R. Hiller, Phys. Rev. D47 (1993) 4647.

[25] D. Kutasov, Phys. Rev. D48 (1993) 4980.

[26] P. Haney, J.R. Hiller, O. Lunin, S. Pinsky, and U. Trittmann Phys. Rev. D62 (2000) 075002, hep-th/9911243.

[27] J.R. Hiller, S. Pinsky, and U. Trittmann, "Two-point stress-tensor correlator in $\mathcal{N}=1,(2+1)$-dimensional super Yang-Mills theory," to appear in Phys. Rev. D, hep-th/0101120. 